International Mathematical Forum, 2, 2007, no. 27, 1329 - 1340

\title{
Fixed Point Theorems for a $k$-set Contraction Map on a Nearly-convex Subset of a Locally Convex Space ${ }^{1}$ \\ Chi-Ming Chen and Cheng-Te Liu \\ Department of Applied Mathematics \\ National Hsin-Chu University of Education, Taiwan, R.O.C.
}

\begin{abstract}
In this paper, the first part, we establish the fixed point theorems for a $k$-set contraction map on the class $Q(X, Y)$, (see [1]). The second part, we generalize the $K K M$ pproperty on a convex set [2] to the $K K M^{*}$ property on a nearly-convex set, and then we establish fixed point theorems, matching theorems and variational inequalities for this class.
\end{abstract}

Mathematics Subject Classification: 47H10, 54C60, 54H25, 55M20

Keywords: nearly-convex set, $k$-set contraction, $K K M^{*}(X, Y), Q(X, Y)$, coincidence theorem, matching theorem, variational inequality

\section{Introduction and Preliminaries}

In 1930, Schauder had shown that a continuous compact map $f: X \rightarrow X$ defined on a closed convex subset $X$ of a Banach space has a fixed point. This theorem enormous influence on fixed point theory, variational inequalities and equilibrium problems. Recently there are appeared some results on fixed point so-called Kakutani factorizable multi-functions defined on convex sets. In this paper, we will invoke nearly-convexity of constraint regions in place of convexity. The first part, we establish the fixed point theorem and coincidence theorem for a $k$-set contraction map on the class $Q(X, Y)$. The second part, we generalize the $K K M$ property on a convex set to the $K K M^{*}$ property on a nearly-convex set, and then we establish the fixed point theorem for a $k$-set contraction map on the family $K K M^{*}(X, X)$, which not need a comapct map.

\footnotetext{
${ }^{1}$ Research supported by the NSC.
} 
Let $X$ and $Y$ be two sets, and let $T: X \rightarrow 2^{Y}$ be a set-valued mapping. We shall use the following notations in the sequel.

(i) $T(x)=\{y \in Y: y \in T(x)\}$,

(ii) $T(A)=\cup_{x \in A} T(x)$,

(iii) $T^{-1}(y)=\{x \in X: y \in T(x)\}$,

(iv) $T^{-1}(B)=\{x \in X: T(x) \cap B \neq \phi\}$,

(v) $T$ is said to lower semicontinuous if for each open subset $B$ of $Y, T^{-1}(B)$ is open in $X$, and

(vi) if $D$ is a nonempty subset of $X$, then $\langle D\rangle$ denote the class of all nonempty finite subset of $D$.

For the case that $X$ and $Y$ are two topological spaces. Then $T$ is said to be closed if its graph $\mathcal{G}_{T}=\{(x, y) \in X \times Y: y \in T(x)\}$ is closed. $T$ is said to be compact if the image $T(X)$ of $X$ under $T$ is contained in a compact subset of $Y$. A subset $D$ of $X$ is said to be compactly closed (resp. compactly open) in $X$ if for any compact subset $K$ of $X$, the set $D \cap K$ is closed (resp. closed) in $K$. Obviously, $D$ is compactly open in $X$ if and only if its complement $D^{c}$ is compactly closed in $X$.

We now introduce a new class of nearly-convex sets. A nonempty subset $X$ of a Hausdorff topological vector space $E$ is said to be nearly-convex [4] if for every compact subset $A$ of $X$ and every neighborhood $V$ of the origin 0 of $E$, there is a continuous mapping $h_{A, V}: A \rightarrow X$ such that $x \in h_{A, V}(x)+V$ for each $x \in A$ and $c o\left(h_{A, V}(A)\right) \subset X$. We call $h_{A, V}$ a continuous convex-inducing mapping.

Remark 1 ( $i)$ In general, the continuous convex-inducing mapping $h_{A, V}$ is not unique. If $U \subset V$, then it is clear that any $h_{A, U}$ can be regarded as an $h_{A, V}$.

(ii) It is clear that the convex set is nearly-convex, but the inverse is not true. For a counterexample,

Let $(M, d)$ be a metric space, $M=\Re^{2}$, we define the metric $d(x, y)=$ $\max \left\{\left|x_{1}-y_{1}\right|,\left|x_{2}-y_{2}\right|\right\}$, where $x=\left(x_{1}, x_{2}\right), y=\left(y_{1}, y_{2}\right) \in M$. Then the set $B(0)=\{x \in M: d(x, 0)<1\} \cup\{(1,0),(-1,0),(0,1)(0,-1)\}$ is a nearly-convex set, but not convex.

Let $E$ will denote a Hausdorff topological vector space, and $B(E)$ the family of nonempty bounded subsets. 
Let $\mathcal{P}=\{P \mid P$ is a family of seminorms which determines the topology on $E\}$. Let $\mathcal{R}^{+}$be the set of all nonnegative real numbers. A mapping $\Phi: B(E) \rightarrow \mathcal{R}^{+}$ is called a measure of noncompactness [3] provided the following conditions hold:

(i) $\Phi(\overline{c o}(\Omega))=\Phi(\Omega)$ for each $\Omega \in B(E)$, where $\overline{c o}(\Omega)$ denotes the closure of the convex hull of $\Omega$,

(ii) $\Phi(\Omega)=0$ if and only if $\Omega$ is precompact,

(iii) $\Phi(A \cup B)=\max \{\Phi(A), \Phi(B)\}$, for each $A, B \in B(E)$, and

(iv) $\Phi(\lambda \Omega)=\lambda \Phi(\Omega)$, for each $\lambda \geq 0, \Omega \in B(E)$.

The above notion is a generalization of the set measure of noncompactness; if $\{p: P\}, P \in \mathcal{P}$ is a family of seminorms which determines the topology on $E$, then for each $p \in P$ and $\Omega \in E$, we define the set-measure of noncompactness $\alpha_{p}: 2^{E} \rightarrow \mathcal{R}^{+}$by

$$
\alpha_{p}(\Omega)=\inf \{\varepsilon>0: \Omega \text { can be covered by a finite number of sets and each }
$$

$$
p \text { - diameter of the sets is less than } \varepsilon\}
$$

where the $p$-diameter of a set $D=\sup \{p(x-y): x, y \in D\}$.

Definition 1 A mapping $T: X \rightarrow 2^{E}$ is said to be a $k$-set contraction map, if there exists $P \in \mathcal{P}$ such that for each $p \in P, \alpha_{p}(T(\Omega)) \leq k \alpha_{p}(\Omega)$ with $k \in(0,1)$ for each bounded subset $\Omega$ of $X$ and $T(X)$ is bounded.

Let $X$ be a subset of a Hausdorff topological vector space $E$ and $Y$ a topological space, we now define a new class $Q(X, Y)$ of the set-valued mappings from $X$ into $Y$ as follows:

$T \in Q(X, Y)$

$\Longleftrightarrow$ for any comapct convex subset $K$ of $X$ and any continuous mapping

$f: T(K) \rightarrow K$, the composition $f\left(\left.T\right|_{K}\right): K \rightarrow K$ has a fixed point.

We next generalize the $K K M$ property (see, [2]) on a convex set of a topological vector space to the following form for a nearly-convex set $X$. 
Definition 2 Let $X$ be a nearly-convex subset of a topological vector space $E$, and $Y$ a topological space. If $T, F: X \rightarrow 2^{Y}$ are two set-valued mappings such that for each finite subset $A$ of $X$ and every neighborhood $V$ of the origin 0 of $E$, there exists a continuous convex-inducing mapping $h_{A, V}: A \rightarrow X$ such that $T\left(\operatorname{co}\left(h_{A, V}(A)\right)\right) \subset F(A)$, then we call $F$ a generalized $K K M^{*}$ mapping with respect to $T$.

Let $T: X \rightarrow 2^{Y}$ be a set-valued mapping such that if $F: X \rightarrow 2^{Y}$ is a generalized $K K M^{*}$ mapping with respect to $T$ then the family $\{\overline{F x}: x \in$ $X\}$ has the finite intersection property, then we say that $T$ has the $K K M^{*}$ property. Denote

$$
K K M^{*}(X, Y)=\left\{T: X \rightarrow 2^{Y} \mid T \text { has the } K K M^{*} \text { property }\right\} .
$$

Remark 2 In particular, for the case $\operatorname{co}(A) \subset X$, we may let the convexinducing mapping $h_{A, V}$ be the identity mapping.

Remark 3 In general, $Q(X, Y)$ and $K K M^{*}(X, Y)$ may not be comparable. (see,[1])

\section{Fixed point theorem for the class $Q(X, Y)$}

In this section, we establish a fixed point theorem for a $k$-set contraction map on the class $Q(X, Y)$, which not need to be a compact map.

Theorem 1 Let $X$ be a nonempty bounded nearly-convex subset of a Hausdorff topological vector space $E$. Assume that $T$ is a $k$-set contraction map, $0<k<$ 1. Then $X$ contains a precompact nearly-convex subset.

Proof, Since $T$ is a $k$-set contraction map, $0<k<1$, there exists $P \in \mathcal{P}$ such that for each $p \in P$, we have $\alpha_{p}(T(A)) \leq k \alpha_{p}(A)$ for each subset $A$ of $X$. Take $y \in X$. Let

$$
\begin{gathered}
X_{0}=X, \quad X_{1}=\operatorname{co}\left(T\left(X_{0}\right) \cup\{y\}\right) \cap X, \text { and } \\
X_{n+1}=\operatorname{co}\left(T\left(X_{n}\right) \cup\{y\}\right) \cap X, \text { for each } n \in N .
\end{gathered}
$$

Then,

(1) $X_{n}$ is nearly-convex, for each $n \in N$,

(2) $X_{n+1} \subset X_{n}$, for each $n \in N$

(3) $T\left(X_{n}\right) \subset X_{n+1}$, for each $n \in N$ and

(4) $\alpha_{p}\left(X_{n+1}\right) \leq \alpha_{p}\left(T\left(X_{n}\right)\right) \leq k \alpha_{p}\left(X_{n}\right) \leq \ldots \leq k^{n+1} \alpha_{p}\left(X_{0}\right)$, for each $n \in N$. 
Thus $\alpha_{p}\left(X_{n}\right) \rightarrow 0$, as $n \rightarrow \infty$, and hence $X_{\infty}=\bigcap_{n \geq 1} X_{n}$ is a nonempty precompact nearly-convex set.

Remark 4 In the process of the proof of Theorem 1, we call the set $X_{\infty} a$ precomapct-inducing nearly-convex subset of $X$.

Corollary 1 Let $X$ be a nonempty bounded convex subset of a Hausdorff topological vector space $E$. Assume that $T$ is a $k$-set contraction map, $0<k<1$. Then $X$ contains a precompact-inducing convex subset.

Theorem 2 Let $X$ be a nonempty bounded nearly-convex subset of a Hausdorff topological vector space $E$, and let $T \in Q(X, X)$. Assume that:

(i) $T$ is a $k$-set contraction map, $0<k<1$ and closed, and

(ii) the precompact-inducing nearly-convex subset $X_{\infty}$ of $X, \overline{T\left(X_{\infty}\right)} \subset X_{\infty}$

Then $T$ has a fixed point in $X$.

Proof, Let $\mathcal{N}=\left\{U_{i}: i \in I\right\}$ be a local base of $E$ such that $U_{i}$ is symmetric and open for each $i \in I$, and let $V \in \mathcal{N}$.

By $i i$, since $\alpha_{p}\left(\overline{T\left(X_{n}\right)}\right) \rightarrow 0$, as $n \rightarrow \infty$, hence $\overline{T\left(X_{\infty}\right)}=\bigcap_{n \geq 1} \overline{T\left(X_{n}\right)}$ is a nonempty compact subset of $X_{\infty}$. And, since $X_{\infty}$ is nearly-convex, there is a continuous mapping $h_{\overline{T\left(X_{\infty}\right)}, V}: \overline{T\left(X_{\infty}\right)} \rightarrow X_{\infty}$ such that $x \in h_{\overline{T\left(X_{\infty}\right)}, V}(x)+V$ for each $x \in \overline{T\left(X_{\infty}\right)}$ and $c o\left(h_{\overline{T\left(X_{\infty}\right)}, V}\left(\overline{T\left(X_{\infty}\right)}\right)\right) \subset X_{\infty}$.

Let $Z=c o\left(h_{T\left(X_{\infty}\right)}, V\left(\overline{T\left(X_{\infty}\right)}\right)\right)$. Then $Z$ is a compact and convex subset of $X$, and $T(Z) \subset X_{\infty}$. Since $T \in Q(X, X)$ and $h_{\overline{T\left(X_{\infty}\right)}, V}$ is continuous, the composition $\left.h_{\overline{T\left(X_{\infty}\right)}, V} \circ T\right|_{Z}: Z \rightarrow 2^{Z}$ has a fixed point, say $x_{V} \in h_{\overline{T\left(X_{\infty}\right)}, V}\left(T\left(x_{V}\right)\right)$. Let $x_{V}=h_{\overline{T\left(X_{\infty}\right)}, V}\left(y_{V}\right)$ for some $y_{V} \in T\left(x_{V}\right) \subset T(Z) \subset \overline{T\left(X_{\infty}\right)}$. Then $y_{V} \in x_{V}+V=h_{\overline{T\left(X_{\infty}\right)}, V}\left(y_{V}\right)+V$. Since $\overline{T\left(X_{\infty}\right)}$ is compact, we may assume that $\left\{y_{V}\right\}$ converges to $\bar{x}$, and then $\left\{x_{V}\right\}$ also converges to $\bar{x}$. The closedness of $T$ implies $\bar{x} \in T(\bar{x})$.

Follow above Theorem 2, we immediate have the following corollary.

Corollary 2 [1] Let $X$ be a nonempty nearly-convex subset of a Hausdorff topological vector space $E$, and let $T \in Q(X, X)$ be compact and closed with $\overline{T(X)} \subset X$. Then $T$ has a fixed point in $X$.

We next establish the following coincidence theorem. 
Theorem 3 Let $X$ be a nonempty bounded convex subset of a Hausdorff topological vector space $E$, and let $T, G: X \rightarrow 2^{X}$ be two set-valued mappings. Assume that:

(i) $T \in Q(X, X)$ is a $k$-set contraction map , $0<k<1$ and closed with $\overline{T(X)} \subset X$

(ii) for each $y \in G(X), G^{-1}(y)$ is convex, and

(iii) for the precompact-inducing convex subset $X_{\infty}$ of $X, \overline{T\left(X_{\infty}\right)} \subset \cup\{\operatorname{int} G(x)$ : $\left.x \in X_{\infty}\right\}$.

Then there exists an $x_{0} \in X$ such that $T\left(x_{0}\right) \cap G\left(x_{0}\right) \neq \phi$.

Proof, By the same process of the proof of Theorem 2, we get a compact subset $\overline{T\left(X_{\infty}\right)}$ of $X$.

By $($ iii $)$, there exists a finite subset $\left\{x_{1}, x_{2}, \ldots, x_{n}\right\}$ of $X_{\infty}$ such that $\overline{T\left(X_{\infty}\right)} \subset$ $\cup_{i=1}^{n} \operatorname{int} G\left(x_{i}\right)$. Let $\left\{\lambda_{i}\right\}_{i=1}^{n}$ be a partition of the unity subordinated to $\left\{\operatorname{int} G\left(x_{i}\right)\right.$ : $i=\{1,2, \ldots, n\}\}$, and let $P=\operatorname{co}\left\{x_{1}, x_{2}, \ldots, x_{n}\right\}$. Define $f: \overline{T\left(X_{\infty}\right)} \rightarrow P$ by

$$
f(y)=\sum_{i=1}^{n} \lambda_{i}(y) x_{i}=\sum_{i \in N_{y}} \lambda_{i}(y) x_{i}, \text { for each } y \in \overline{T\left(X_{\infty}\right)}
$$

where $i \in N_{y}$ iff $\lambda_{i}(y) \neq 0$ iff $y \in \operatorname{int} G\left(x_{i}\right) \subset G\left(x_{i}\right)$,

Then $x_{i} \in G^{-1}(y)$ for each $i \in N_{y}$. Clearly, $f$ is continuous, and by $(i i)$, we have

$$
f(y) \in \operatorname{co}\left\{x_{i}: i \in N_{y}\right\} \subset G^{-1}(y) \text {, for each } y \in \overline{T\left(X_{\infty}\right)} .
$$

Since $P$ is compact convex subset of $X$ and $T \in Q(X, X)$, the composition $f|T(P) \circ T|_{P}: P \rightarrow P$ has a fixed point $x_{0} \in P \subset X$. So $x_{0} \in f T\left(x_{0}\right)$ and $f^{-1}\left(x_{0}\right) \subset G\left(x_{0}\right)$. So, we complete the proof.

\section{$3 \quad$ Fixed point theorem for the class $K K M^{*}(X, Y)$}

In this section, we establish the fixed point theorem for a $k$-set contraction map on the class $K K M^{*}(X, Y)$, which not need to be a compact map.

The following Lemma 1 will play important role for this paper.

Lemma 1 Let $X$ be a nonempty nearly-convex subset of a Hausdorff topological vector space $E, Y$ a topological space. Then $\left.T\right|_{D} \in K K M^{*}(D, Y)$ whenever $T \in K K M^{*}(X, Y)$ and $D$ is a nonempty nearly-convex subset of $X$. 
Proof, Let $F: D \rightarrow 2^{Y}$ be a generalized $K K M^{*}$ mapping with respect to $\left.T\right|_{D}$. Then for any finite subset $A$ of $D$ and any neighborhood $V$ of the origin 0 of $E$, there exists a continuous convex-inducing mapping $h_{A, V}: A \rightarrow D$ such that $\left.T\right|_{D}\left(c o\left(h_{A, V}(A)\right)\right) \subset F(A)$.

Define $F^{\prime}: X \rightarrow 2^{Y}$ by

$$
F^{\prime}(x)= \begin{cases}F(x) & x \in D, \\ Y & x \in X \backslash D .\end{cases}
$$

It is clear that for any finite subset $B$ of $X$ and any any neighborhood $U$ of the origin 0 of $E$, there exists a continuous convex-inducing mapping $h_{B, U}: B \rightarrow X$ such that $T\left(c o\left(h_{B, U}(B)\right)\right) \subset F^{\prime}(B)$. Indeed,

(1) if $B \nsubseteq D$, then there exists some $x \in B \backslash D$, and hence $F^{\prime}(x)=Y$. So the result is obvious.

(2) if $B \subset D$, since $F$ be a generalized $K K M^{*}$ mapping with respect to $\left.T\right|_{D}$, the inclusion is true.

Thus $F^{\prime}$ is a generalized $K K M^{*}$ mapping with respect to $T$. Since $T \in$ $K K M^{*}(X, Y)$, hence the family $\left\{\overline{F^{\prime} x}: x \in X\right\}$ has finite intersection property, and so does the family $\{\overline{F x}: x \in D\}$. So $\left.T\right|_{D} \in K K M^{*}(D, Y)$.

Lemma 2 Let $X$ be a nonempty nearly-convex subset of a Hausdorff topological vector space $E, Y$ and $z$ are two topological spaces. Then $f T \in K K M^{*}(X, Z)$ whenever $T \in K K M^{*}(X, Y)$ and $f \in C(Y, Z)$.

Proof, Let $F$ be a generalized $K K M^{*}$ mapping with respect to $f T$. Then for any $A=\left\{x_{1}, x_{2}, \ldots, x_{n}\right\} \in\langle X\rangle$ and any neighborhood of the origin 0 of $E$, there exists a continuous convex-inducing mapping $h_{A, V}(A): A \rightarrow X$ such that $f T\left(\operatorname{co}\left(h_{A, V}(A)\right)\right) \subset \cup_{i=1}^{n} F\left(x_{i}\right)$. So, $T\left(c o\left(h_{A, V}(A)\right)\right) \subset \cup_{i=1}^{n} f^{-1} F\left(x_{i}\right)$, which says that $f^{-1} F$ is a generalized $K K M^{*}$ mapping with respect to $T$. Since $T \in K K M^{*}(X, Y)$, the family $\left\{f^{-1} F(x): x \in X\right\}$ has the finite intersection property, and so does the family $\{F(x): x \in X\}$. This shows that $f T \in$ $K K M^{*}(X, Z)$.

Theorem 4 Let $X$ be a nonempty bounded nearly-convex subset of a locally convex space $E$, and let $T \in K K M^{*}(X, X)$ is a $k$-set contraction, $0<k<1$ and closed with $\overline{T(X)} \subset X$. Then $T$ has a fixed point in $X$.

Proof, Let $\mathcal{N}=\left\{U_{i}: i \in I\right\}$ be a local base of $E$ such that $U_{i}$ is symmetric, open and convex for each $i \in I$, and let $V \in \mathcal{N}$.

And, by the same process of the proof of Theorem 2, we get a compact subset $\overline{T\left(X_{\infty}\right)}$ of $X$. Since $\overline{T\left(X_{\infty}\right)} \subset \overline{T(X)} \subset X$ and $X$ is nearly-convex, there 
is a continuous mapping $h_{\overline{T\left(X_{\infty}\right)}, V}: \overline{T\left(X_{\infty}\right)} \rightarrow X$ such that $x \in h_{\overline{T\left(X_{\infty}\right)}, V}(x)+V$ for each $x \in \overline{T\left(X_{\infty}\right)}$ and $c o\left(h_{\overline{T\left(X_{\infty}\right)}, V}\left(\overline{T\left(X_{\infty}\right)}\right)\right) \subset X$.

Let $Z=c o\left(h_{\overline{T\left(X_{\infty}\right)}, V}\left(\overline{T\left(X_{\infty}\right)}\right)\right)$. Then $Z$ is a compact and convex subset of $X$, and $T(Z) \subset X$. Since $T \in K K M^{*}(X, X)$ and $X_{\infty}$ is a nearly-convex subset of $X$, by Lemma 1 , we have $\left.T\right|_{X_{\infty}} \in K K M^{*}\left(X_{\infty}, X\right)$. Next, since $h_{\overline{T\left(X_{\infty}\right)}, V}$ is continuous, by Lemma 2, we have $\left.h_{\overline{T\left(X_{\infty}\right)}, V} \circ T\right|_{X \infty} \in K K M^{*}\left(X_{\infty}, Z\right)$. Put

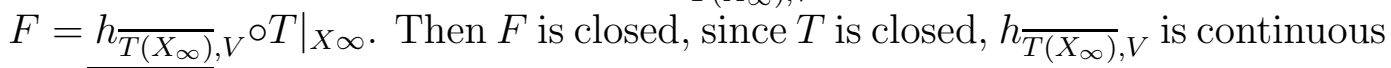
and $\overline{T\left(X_{\infty}\right)}$ is compact.

We now claim that for each $U_{i} \in \mathcal{N}$, there exists an $x_{i} \in X_{\infty}$ such that

$$
\left(x_{i}+U_{i}+V\right) \cap F\left(x_{i}\right) \neq \phi .
$$

If the above statesment is not true, then there exists $U \in \mathcal{N}$ such that $(x+$ $U+V) \cap F(x)=\phi$, for all $x \in X_{\infty}$.

Let $K=\overline{h_{\overline{T\left(X_{\infty}\right)}, V}\left(T\left(X_{\infty}\right)\right)}$. Then $K \subset h_{\overline{T\left(X_{\infty}\right)}, V}\left(\overline{T\left(X_{\infty}\right)}\right) \subset Z$. Define $G: X_{\infty} \rightarrow 2^{Z}$ by

$$
G(x)=K \backslash\left(x+\frac{1}{2} U+V\right), \text { for each } x \in X_{\infty} .
$$

Then

(1) $G(x)$ is compact, for each $x \in X_{\infty}$, and

(2) $G$ is a generalized $K K M^{*}$ mapping with respect to $F$.

We prove (2) by contradiction. Let $A=\left\{x_{1}, x_{2}, \ldots, x_{n}\right\} \subset X_{\infty}$. Then there exists $V^{\prime} \in \mathcal{N}$ such that for any continuous convex-inducing $h_{A, V^{\prime}}: A \rightarrow X_{\infty}$ one has $F\left(c o\left(h_{A, V^{\prime}}(A)\right)\right) \nsubseteq G(A)$. Let $U^{\prime} \in \mathcal{N}$ such that $U^{\prime} \subset \frac{1}{2} U \cap V^{\prime}$. Then $F\left(c o\left(h_{A, U^{\prime}}(A)\right)\right) \nsubseteq G(A)$. So, there is $u \in c o\left(h_{A, U^{\prime}}(A)\right)$ and $v \in F(u)$ such that $v \notin \cup_{i=1}^{n} G\left(x_{i}\right)$. From the definition of $G$, it follows that $v \in\left(x_{i}+\frac{1}{2} U+V\right)$, for each $i \in\{1,2, \ldots, n\}$. Hence $v \in\left(x_{i}+\frac{1}{2} U+V\right) \in\left(h_{A, U^{\prime}}\left(x_{i}\right)+\frac{1}{2} U+\right.$ $\left.V+U^{\prime}\right) \subset\left(h_{A, U^{\prime}}\left(x_{i}\right)+U+V\right)$, for each $i \in\{1,2, \ldots, n\}$, since $X_{\infty}$ is nearlyconvex. Thus, $h_{A, U^{\prime}}\left(x_{i}\right) \in(v+U+V)$, for each $i \in\{1,2, \ldots, n\}$, and hence $u \in c o\left(h_{A, U^{\prime}}(A)\right) \in v+U+V$. So, $v \in u+U+V$ and $v \in F(u)$, we conclude that $F(u) \cap(u+U+V) \neq \phi$, a contradiction. Therefore, $G$ is a generalized $K K M^{*}$ mapping with respect to $F$.

Since $F \in K K M^{*}\left(X_{\infty}, Z\right)$ and $G$ is a generalized $K K M^{*}$ mapping with respect to $F$, the family $\left\{G(x): x \in X_{\infty}\right\}$ has the finite intersection property, and so we conclude that $\cap_{x \in X_{\infty}} G(x) \neq \phi$. Choose $\eta \in \cap_{x \in X_{\infty}} G(x)$, then $\eta \in K \backslash\left(x+\frac{1}{2} U+V\right)$, for each $x \in X_{\infty}$. Since $\eta \in \cap_{x \in X_{\infty}} G(x) \subset K \subset$ $h_{\overline{T\left(X_{\infty}\right)}, V}\left(\overline{T\left(X_{\infty}\right)}\right) \subset \overline{T\left(X_{\infty}\right)}+V \subset \overline{X_{\infty}}+V$, hence there is an $x_{0} \in X_{\infty}$ such that $\eta \in x_{0}+\frac{1}{2} U+V$. But $\eta \in K \backslash\left(x_{o}+\frac{1}{2} U+V\right)$, a contradiction. Therefore, we have proved for each $U_{i} \in \mathcal{N}$, there exists an $x_{i} \in X_{\infty}$ such that 
$\left(x_{i}+U_{i}+V\right) \cap F\left(x_{i}\right) \neq \phi$. Let $y_{i} \in\left(x_{i}+U_{i}+V\right) \cap F\left(x_{i}\right)$. Since $\left\{y_{i}\right\} \subset K$ and $K$ is comapct, we may assume that $\left\{y_{i}\right\}$ converges to some $y_{0} \in K$, and since $\left\{x_{i}\right\} \subset \overline{X_{\infty}}$, we assume that $\left\{x_{i}\right\}$ converges to $x_{0}$. The closedness of $F$ implies that $\left(x_{0}, y_{0}\right) \in \mathcal{G}_{F}$, so we have $y_{0} \in x_{0}+\bar{V}$ and $y_{0} \in F\left(x_{0}\right)=$ $h_{\overline{T\left(X_{\infty}\right)}, V}\left(T\left(x_{0}\right)\right)$. Choose $z_{0} \in T\left(x_{0}\right)$ such that $y_{0}=h_{\overline{T\left(X_{\infty}\right)}, V}\left(z_{0}\right)$. Noting that $z_{0} \in h \overline{T\left(X_{\infty}\right)}, V\left(z_{0}\right)+V=y_{0}+V \subset x_{0}+V+\bar{V} \subset\left(x_{0}+V+V+V\right)$, and hence $T\left(x_{0}\right) \cap x_{0}+V+V+V \neq \phi$ for any $V \in \mathcal{N}$, which just as before, implies $T$ has a fixed point.

Corollary 3 Let $X$ be a nonempty bounded convex subset of a locally convex space $E$, and let $T \in K K M(X, X)$ is a k-set contraction, $0<k<1$ and closed with $\overline{T(X)} \subset X$. Then $T$ has a fixed point in $X$.

Corollary 4 Let $X$ be a nonempty nearly-convex subset of a locally convex space $E$, and let $T \in K K M^{*}(X, X)$ is compact and closed. Then $T$ has a fixed point in $X$.

Corollary 5 [2] Let $X$ be a nonempty convex subset of a locally convex space $E$, and let $T \in K K M(X, X)$ is compact and closed. Then $T$ has a fixed point in $X$.

We now establish the following $K K M^{*}$-type theorem, which is equivalent to the matching theorem after it.

Theorem 5 Let $X$ be a nonempty bounded nearly-convex subset of a Hausdorff topological vector space $E$. If $T, F: X \rightarrow 2^{X}$ are two set-valued mappings satisfying the following:

(i) $T \in K K M^{*}(X, X)$ is a $k$-set contraction map, $0<k<1$ with $\overline{T(X)} \subset$ $X$,

(ii) for any $x \in X, F(x)$ is compactly closed in $X$, and

(iii) $F$ is a generalized $K K M^{*}$ mapping with respect to $T$,

then

$$
\overline{T\left(X_{\infty}\right)} \cap\left(\cap\left\{F(x): x \in X_{\infty}\right\}\right) \neq \phi,
$$

where $X_{\infty}$ is the precompact-inducing nearly-convex subset of $X$. 
Proof, Let $\mathcal{N}=\left\{U_{i}: i \in I\right\}$ be a local base of $E$ such that $U_{i}$ is symmetric and open for each $i \in I$. By the same process of the proof of Theorem 2, we get a compact subset $\overline{T\left(X_{\infty}\right)}$ of $X$, and $\left.T\right|_{X_{\infty}} \in K K M^{*}\left(X_{\infty}, X\right)$, since $T \in K K M^{*}(X, X)$.

Define $H: X_{\infty} \rightarrow 2^{X}$ by

$$
H(x)=\overline{T\left(X_{\infty}\right)} \cap F(x), \text { for each } x \in X_{\infty} .
$$

It follows from $(\mathrm{iii})$ that $F$ is a generalized $K K M^{*}$ mapping with respect to $\left.T\right|_{X_{\infty}}$, and hence for any $x \in X_{\infty}$ and any $V \in \mathcal{N}$, there exists a continuous convex-inducing $h_{\{x\}, V}:\{x\} \rightarrow X_{\infty}$ such that $T\left(\operatorname{co}\left(h_{\{x\}, V}(\{x\})\right)\right) \subset F(x)$ and $\operatorname{co}\left(h_{\{x\}, V}(x)\right) \subset X_{\infty}$. So $H(x) \neq \phi$.

By $(i i), H(x)$ is compact in $X$, for each $x \in X_{\infty}$. We now claim that $H$ is a generalized $K K M^{*}$ mapping with respect to $\left.T\right|_{X_{\infty}}$. Let $A \in\left\langle X_{\infty}\right\rangle$. By (iii), for any $V \in \mathcal{N}$, there exists a continuous convex-inducing mapping $h_{A, V}: A \rightarrow X_{\infty}$ such that $T\left(c o\left(h_{A, V}(A)\right)\right) \subset F(A)$ and $\operatorname{co}\left(h_{A, V}(A)\right) \subset X_{\infty}$. So, $T\left(c o\left(h_{A, V}(A)\right)\right) \subset F(A) \cap \overline{T\left(X_{\infty}\right)}=H(A)$. Thus, we have shown that $H$ is a generalized $K K M^{*}$ mapping with respect to $\left.T\right|_{X_{\infty}}$. Since $\left.T\right|_{X_{\infty}} \in$ $K K M^{*}\left(X_{\infty}, X\right)$, the family $\left\{H(x): x \in X_{\infty}\right\}$ has the finite intersection property. And, since $H(x)$ is compact, hence $\cap_{x \in X_{\infty}} H(x) \neq \phi$, that is, $\overline{T\left(X_{\infty}\right)} \cap\left(\cap\left\{F(x): x \in X_{\infty}\right\}\right) \neq \phi$.

Corollary 6 Let $X$ be a nonempty bounded convex subset of a Hausdorff topological vector space $E$. If $T, F: X \rightarrow 2^{X}$ are two set-valued mappings satisfying the following:

(i) $T \in K K M(X, X)$ is a k-set contraction map, $0<k<1$ with $\overline{T(X)} \subset X$,

(ii) for any $x \in X, F(x)$ is compactly closed in $X$, and

(iii) $F$ is a generalized KKM mapping with respect to $T$,

then there exists a nonempty convex subset $Y$ of $X$ such that

$$
\overline{T\left(X_{\infty}\right)} \cap\left(\cap\left\{F(x): x \in X_{\infty}\right\}\right) \neq \phi,
$$

where $X_{\infty}$ is the precompact-inducing nearly-convex subset of $X$.

As a consequence of the above theorems, we get the following generalization of the Ky Fan matching theorem.

Theorem 6 Let $X$ be a nonempty bounded nearly-convex subset of a Hausdorff topological vector space $E$. If $T, H: X \rightarrow 2^{X}$ are two set-valued mappings satisfying the following: 
(i) $T \in K K M^{*}(X, X)$ is a $k$-set contraction map, $0<k<1$ with $\overline{T(X)} \subset$ $X$,

(ii) for any $x \in X, H(x)$ is compactly open in $X$, and

(iii) for the precompact-inducing nearly-convex subset $X_{\infty}$ of $X, \overline{T\left(X_{\infty}\right)} \subset$ $H\left(X_{\infty}\right)$,

then the precompact-inducing nearly-convex subset $X_{\infty}$ of $X$ satisfies the following condition:

$$
T\left(X_{\infty}\right) \cap(\cap\{H(x): x \in M\}) \neq \phi, \text { for some } M \in\left\langle X_{\infty}\right\rangle .
$$

Proof, Let $\mathcal{N}=\left\{U_{i}: i \in I\right\}$ be a local base of $E$ such that $U_{i}$ is symmetric and open for each $i \in I$. And, by the same process of the proof of Theorem 2, we get a compact subset $\overline{T\left(X_{\infty}\right)}$ of $X$, and $\left.T\right|_{X_{\infty}} \in K K M^{*}\left(X_{\infty}, X\right)$, since $T \in K K M^{*}(X, X)$.

We claim that there exists $M \in\left\langle X_{\infty}\right\rangle$ such that $T\left(X_{\infty}\right) \cap(\cap\{H(x): x \in$ $M\}) \neq \phi$. On the contrary, assume that $T\left(X_{\infty}\right) \cap(\cap\{H(x): x \in M\})=\phi$ for any $M \in\left\langle X_{\infty}\right\rangle$, then $T\left(X_{\infty}\right) \subset \cap_{x \in M} H^{c}(x)$. Since $X_{\infty}$ is nearly-convex, hence for any $V \in \mathcal{N}$, there exists a continuous convex-inducing mapping $h_{M, V}: M \rightarrow X_{\infty}$ such that $c o\left(h_{M, V}(M)\right) \subset X_{\infty}$. So $T\left(\operatorname{co}\left(h_{M, V}(M)\right)\right) \subset$ $T\left(X_{\infty}\right) \subset \cap_{x \in M} H^{c}(x)$. This implies $H^{c}$ is a generalized $K K M^{*}$ mapping with respect to $T$. By $(i i)$, for any $x \in X, H^{c}(x)$ is compaclty closed in $X$. Follows Theorem 5, we have $\overline{T\left(X_{\infty}\right)} \cap\left(\cap\left\{H^{c}(x): x \in X_{\infty}\right\}\right) \neq \phi$, which implies $\overline{T\left(X_{\infty}\right)} \nsubseteq \cup_{x \in X_{\infty}} H(x)$, a contradiction to $(i i i)$. We complete the proof.

Corollary 7 Let $X$ be a nonempty bounded convex subset of a Hausdorff topological vector space $E$. If T, $H: X \rightarrow 2^{X}$ are two set-valued mappings satisfying the following:

(i) $T \in K K M(X, X)$ is a $k$-set contraction map, $0<k<1$ with $\overline{T(X)} \subset X$,

(ii) for any $x \in X, H(x)$ is compactly open in $X$, and

(iii) for the precompact-inducing convex subset $X_{\infty}$ of $X, \overline{T\left(X_{\infty}\right)} \subset H\left(X_{\infty}\right)$, then the precompact-inducing convex subset $X_{\infty}$ of $X$ satisfies the following condition:

$$
T\left(X_{\infty}\right) \cap(\cap\{H(x): x \in M\}) \neq \phi, \text { for some } M \in\left\langle X_{\infty}\right\rangle .
$$

As a consequence of the above Corollary 6, we have the following generalized variational inequality.

Theorem 7 Let $X$ be a nonempty bounded convex subset of a Hausdorff topological vector space $E$, and let $T \in K K M^{*}(X, X)$ be a $k$-set contraction map, $0<k<1$ with $\overline{T(X)} \subset X$. If $\varphi, \psi: X \times X \rightarrow(-\infty, \infty)$ are two real-valued mappings satisfying the following: 
(i) $\psi(x, y) \leq 0$, for each $(x, y) \in \mathcal{G}_{T}$,

(ii) for fixed $x \in X$, the mapping $y \mapsto \varphi(x, y)$ is lower semicontinuous on $K$ for each compact subset $K$ of $X$, and

(iii) for fixed $y \in X$, the set $\{x \in X: \psi(x, y)>0\}$ contains the convex hull of the set $\{x \in X: \varphi(x, y)>0\}$

then for the precompact-inducing convex subset $X_{\infty}$ of $X$, there exists an $\bar{y} \in$ $X_{\infty}$ such that

$$
\varphi(x, \bar{y}) \leq 0 \text { for each } x \in X_{\infty}
$$

Proof, Define $F, S: X \rightarrow 2^{X}$ by

$$
\begin{gathered}
S(x)=\{y \in X: \psi(x, y) \leq 0\} \text { for each } x \in X, \text { and } \\
F(x)=\{y \in X: \varphi(x, y) \leq 0\} \text { for each } x \in X .
\end{gathered}
$$

By assumption $(i)$, we have $\mathcal{G}_{T} \subset \mathcal{G}_{S}$, and by assumption $(i i), F(x)$ is comapctly closed for each $x \in X$. The condition (iii) implies that for each finite subset $A$ of $X, S(\operatorname{co}(A)) \subset F(A)$, and then $T(\operatorname{co}(A)) \subset F(A)$, that is; $F$ is a generalized $K K M$ mapping with respect to $T$.

So, all the conditions in Corollary 6 are satisfied, and so for the precompactinducing convex subset $X_{\infty}$ of $X$, we have that $\overline{T\left(X_{\infty}\right)} \cap\left(\cap\left\{F(x): x \in X_{\infty}\right\}\right) \neq$ $\phi$. Let $\bar{y} \in \overline{T(Y)} \cap(\cap\{F(x): x \in Y\})$, and hence we have $\varphi(x, \bar{y}) \leq 0$ for each $x \in Y$

\section{References}

[1] C. M. Chen, C. L. Yen, Fixed point theorems for the class Q(X,Y), International Journal of Mathematics and Mathematical Science(2005), In press.

[2] T. H. Chang, C. L. Yen, KKM property and fixed point theorems, J. Math. Ann. Appl. 203(1996), 224-235.

[3] P.M.Fitzpatrick, W.V.Petryshyn, Fixed point theorems for multivalued noncompact inward mappings, J. Math. Anal. Appl. 46(1974), 756-767.

[4] T. Y. Wu, General vector quasi-variational inequalities on non-convex contraint regions, In press.

\section{Received: September 4, 2006}

